\title{
Arthroscopic treatment of early glenohumeral arthritis
}

\author{
Giuseppe Porcellini · Giovanni Merolla • \\ Fabrizio Campi • Andrea Pellegrini · \\ Chandra Sekhar Bodanki · Paolo Paladini
}

Received: 17 July 2012/Accepted: 1 November 2012/Published online: 22 November 2012

(C) The Author(s) 2012. This article is published with open access at Springerlink.com

\begin{abstract}
Background The articular cartilage of the shoulder is not endowed with intrinsic repair abilities, so the detection of chondral lesions during arthroscopy may indicate that additional articular procedures are needed. The aim of the current study was to evaluate the benefits of arthroscopy in patients with early shoulder arthritis, and to assess which clinical and radiological features are correlated with better arthroscopic outcomes.

Materials and methods Out of a total of 2,707 shoulders, 61 arthroscopies were performed on patients aged $30-55$ years suffering from a painful early arthritic shoulder. We performed a retrospective study of 47 of those 61 patients with osteoarthritis at Samilson-Prieto stage I or II. SST and Constant score were used as outcome measures. Arthroscopic circumferential capsulotomy was performed to release the soft tissues and increase the joint space. Glenoid chondral lesions were caregorized according to location (anterior, posterior, centered) and size (small, large, total) and treated with microfractures; in the last 11 patients, we placed a engineered hyaluronic acid membrane, Hyalograft ${ }^{\circledR} \mathrm{C}$, on the surface of the glenoid. Postoperative care included mobilization the day after surgery, with the arm protected in a sling for two weeks. Follow-up examinations were performed at 3,6,12, and 24 months after surgery. The clinical and radiographic data collected were compared with those obtained at the last examination. Results The mean Constant score increased from 43.8 points to 79.1, and the mean SST score increased from 4.9
\end{abstract}

G. Porcellini · G. Merolla ( $₫)$ · F. Campi · A. Pellegrini · C. S. Bodanki - P. Paladini

Unit of Shoulder and Elbow Surgery, D. Cervesi Hospital,

Via L. Van Beethoven 1, CAP, 47841 Cattolica, RN, Italy

e-mail: giovannimerolla@hotmail.com points to 9.4 points. Clinical outcomes improved significantly in 44 patients $(93.6 \%)$. The three patients $(6.4 \%)$ with the lowest scores showed progression of arthritis. Age, gender, glenohumeral distance, and presence of engineered hyaluronic acid membrane were not related to clinical scores. Recovery of range of motion as well as small and centered cartilage lesions were statistically associated with improved outcome.

Conclusion The main finding was that soft tissue procedures (including capsulotomy and synovectomy) associated with glenoid microfractures are only suitable for patients with early arthritis and preserved humeral head shape, particularly in cases with small and centered glenoid cartilage lesions.

Keywords Early arthritis - Shoulder · Arthroscopy · Cartilage lesions

\section{Introduction}

The articular cartilage of the shoulder is not endowed with intrinsic repair abilities; therefore, when a disease such as instability or cuff injury is present, even minor lesions can rapidly lead to early glenohumeral joint arthritis. Cartilage lesions are not unusual, even in young patients [1], and are often found during arthroscopic procedures performed when such patients have various pathologic conditions [2-4]. Less common conditions include glenoid dysplasia and osteochondritis dissecans [5]. The varying thickness of joint cartilage and resistance properties of the subchondral bone [6] result in lesions with different depths and widths, depending on the resistance offered by the articular surface $[7,8]$. Minor cartilage lesions associated with rotator cuff or glenohumeral ligament damage will induce topographically 
different stresses on the various areas of the articular surface. Recent and older research findings have shown a correlations between cartilage wear and lesion site and between site and symptoms in the shoulder as well as in the knee [9-12]. Several conservative options are available to manage shoulder arthritis: alleviate pain, reduce inflammation, and (especially) halt or at least slow down the evolution of arthritis [13]. Such therapies entail changes in lifestyle as well as systemic and topical drug administration. Viscosupplementation using hyaluronic acid may be a useful treatment option in patients who have shoulder osteoarthritis with an intact rotator cuff [14], while less satisfactory results have been obtained in those with rotator cuff tears or advanced osteoarthritis [15]. Several surgical options are available to manage primary shoulder arthritis, including simple arthroscopic joint debridement [16] and more complex techniques such as resurfacing using fascia lata or meniscus [17], osteochondral autologous transplantation [18], resurfacing arthroplasty [19], and total arthroplasty [20]. The use of microfractures to treat full-thickness chondral defects is a viable option that provides good results in young patients, with the greatest improvements seen for smaller lesions of the humerus and the worst results observed in patients with bipolar lesions [21], even when the microfracture is covered with a periosteal flap [22]. The microfracture technique enhances chondral resurfacing by providing a suitable environment for new tissue formation and taking advantage of the body's own healing potential [23]. A combination of microfractures and viscosupplementation with three weekly injections of intraarticular hyaluronic acid was seen to have positive effects on the repair tissue that formed within the chondral defect at an early follow-up examination: it had possible chondroprotective and anti-inflammatory effects and limited the development of degenerative changes within the joint [24]. The use of an engineered hyaluronic acid membrane gave good results in pilot studies in the knee, whether using the scaffold alone or the scaffold loaded with autologous chondrocytes [25]. The aim of the current study was to evaluate the benefits of arthroscopy in patients with early shoulder arthritis and to assess which clinical and radiological features are correlated with better arthroscopic outcomes.

\section{Materials and methods}

All patients gave informed consent prior to being included in the study. This was a retrospective study that was authorized by the local ethical committee and performed in accordance with the Ethical Standards of the 1964 Declaration of Helsinki as revised in 2000.

Out of a total of 2,707 shoulder procedures performed from January 2006 to December 2008, 61 (2.25\%) arthroscopies were performed on patients aged 30 to 55 years (mean 41.7 ) suffering from a painful early arthritic shoulder. The patients were males in 45 cases $(73.8 \%)$ and females in 16 cases $(26.2 \%)$. A single surgeon performed arthroscopic surgery using a similar arthroscopic technique in all patients. All patients had a preoperative imaging study with X-ray evaluation of the shoulders, leading to classification according to the Samilson and Prieto scheme [26]. Arthritis was grade I or II in all cases. An additional MRI was performed to image the cartilaginous defects on both surfaces. SST and Constant score were used as outcome measures [27, 28]. The treatment approach was selected on the basis of clinical history and imaging data.

Inclusion criteria were: arthritis at Samilson-Prieto stage I or II, passive stiffness $<40^{\circ}$ in forward flexion and $<30^{\circ}$ in external rotation with the arm at the side. During arthroscopy, cartilaginous defects of the glenoid were classified as small $\left(<2 \mathrm{~cm}^{2}\right)$, big $\left(>2 \mathrm{~cm}^{2}\right)$, or total (the defect covers the whole surface). All the glenoid cartilage defects were grade IV [29, 30] or ICRS grade 4a/b [11]. Arthroscopic examination of the humeral head showed that cartilage was still present and the humeral head shape had been maintained. Patients with broad and deep humeral cartilage defects and a squared head were excluded from the study. Of the 61 shoulders treated for painful early arthritis, $50(82 \%)$ met the inclusion criteria. Since 3 (6\%) patients were lost to follow-up, the study was conducted on 47 patients (94\%)-males in 35 cases $(74.5 \%)$ and females in 12 cases $(25.5 \%)$.

Exclusion criteria were passive shoulder stiffness with a loss of forward elevation of $>40^{\circ}$ and a loss of external rotation of $>30^{\circ}$, previous surgery, nerve palsy, and rotator cuff tears.

\section{Radiographic evaluation}

Preoperative X-ray imaging was used to calculate the distance between the glenoid and the humeral head surface.

Radiographic examination was executed as follows. An anteroposterior radiograph in neutral shoulder rotation with the patient standing, a scapular lateral (outlet) radiograph, and an axillary view were obtained at the final follow-up. The articular space was evaluated preoperatively and at the last follow-up by measuring the distance between the glenoid and the humeral head surface on the axillary radiographs [30]. All measurements were performed using OsiriX imaging software (v.3.7.1).

Surgical technique

Patients were placed in lateral decubitus with $5 \mathrm{~kg}$ of traction. Three routine arthroscopic portals (anterior-superior, anterior-inferior, and posterior) were used to perform the 


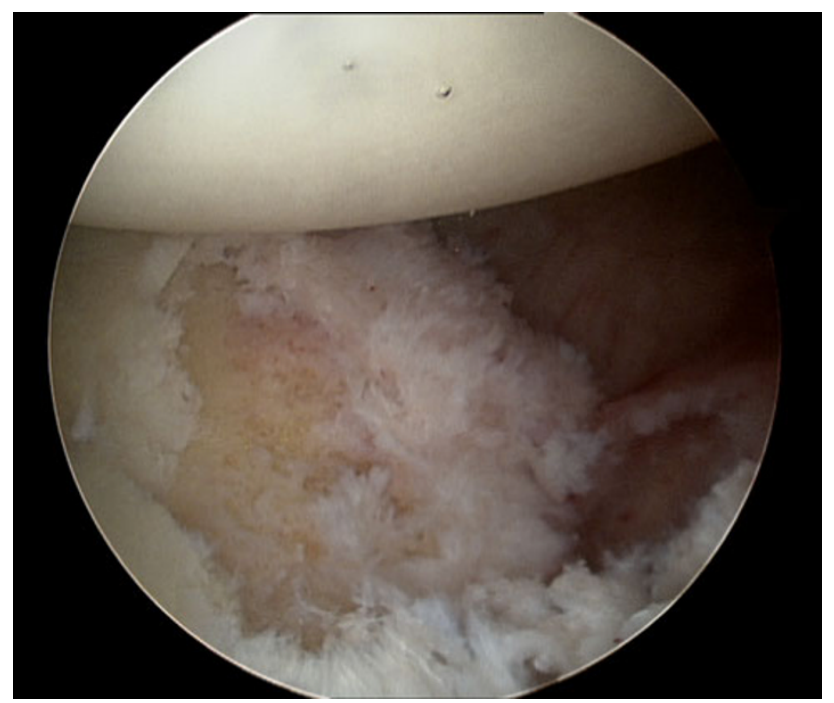

Fig. 1 Glenoid surface after delineating the boundaries of the cartilage lesions

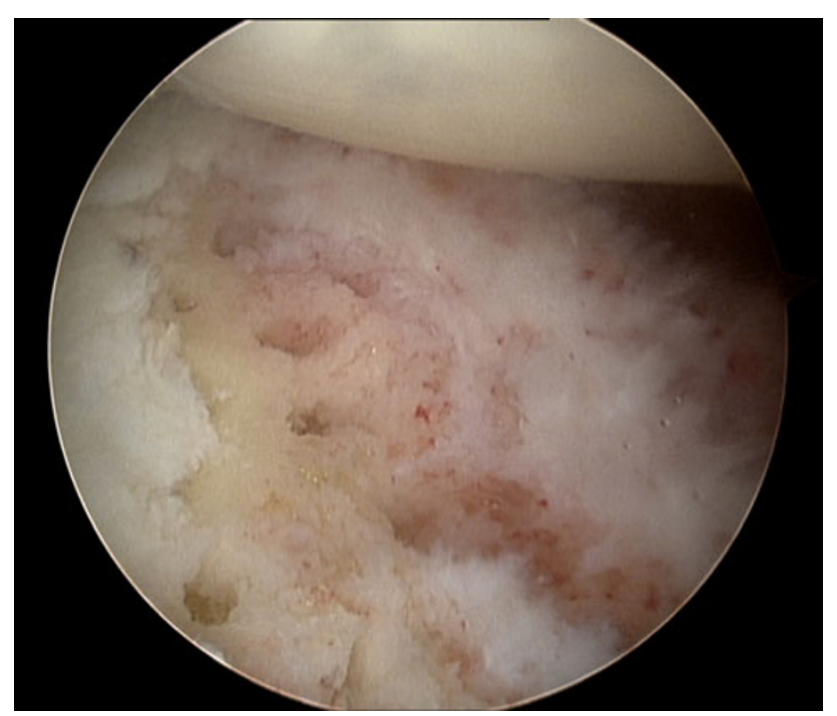

Fig. 2 Glenoid surface after performing microfracture

surgical technique. After initially removing the synovial membrane, a circumferential capsulotomy was performed to achieve release the soft tissues in order to increase the joint space. Rotator interval debridement with removal of hypertrophic synovitis was performed in all cases. Loose bodies were removed if present. After delineating the boundaries of the cartilage lesions (Fig. 1) or of the whole glenoid (leaving the glenoid labrum in situ) and debriding the calcified chondral layer (until punctate bleeding was observed), we implemented microfractures, placing the awl holes at appropriate positions perpendicular to the subchondral plate at 2-3 mm intervals [23] (Fig. 2). For the final $11(23.4 \%)$ patients, after performing the microfractures, we placed an engineered hyaluronic acid membrane

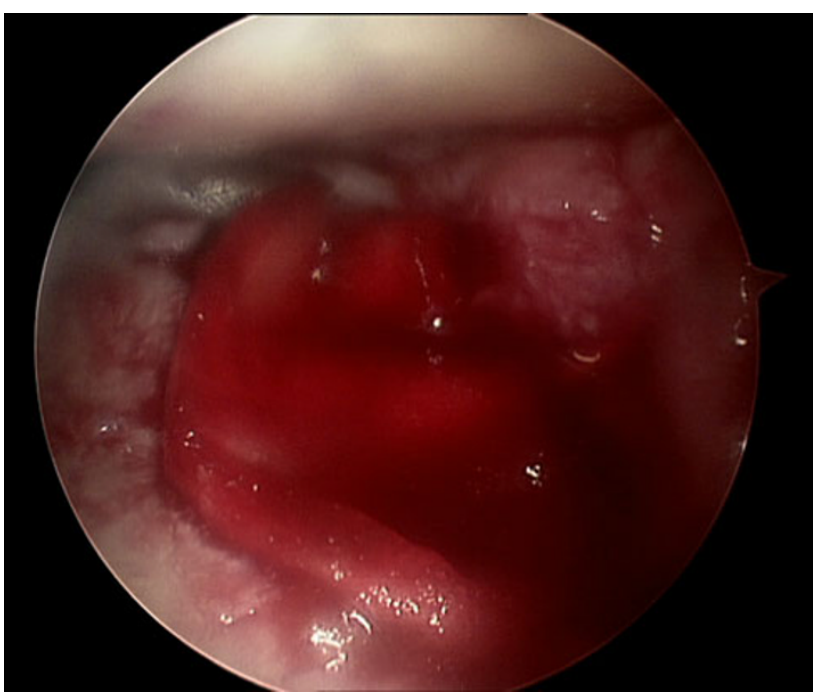

Fig. 3 Engineered hyaluronic acid membrane lying on the glenoid surface without fluid irrigation and traction

(Hyalofast ${ }^{\circledR}$, Fidia Advanced Biopolymers S.r.1., Abano Terme, Italy) on the glenoid surface. The membrane was first cut into the shape of the glenoid chondral lesion and placed without using fixation devices such as screws or fibrin glue. The placement of the membrane was achieved by passing it through a $8.5 \mathrm{~mm}$ cannula in the anteriorinferior portal. A global inspection of the joint, without fluid irrigation and traction (Fig. 3), was performed at the end of the procedure in order to evaluate the stability of the membrane during humeral head movement.

\section{Postoperative rehabilitation}

The rehabilitation program simply involved the use of a sling for the first two weeks after surgery. Immediate passive mobilization began the day after the operation, under the supervision of a physiotherapist. Pool exercises and active assisted exercises within the pain-free range of motion were started three weeks after surgery. Active exercises to balance the internal and external rotators of the shoulder with a rubber band were initiated after eight weeks. Additionally, for all patients, physical therapy was performed in our institution's outpatient rehabilitation unit for about six months to strengthen the shoulder and maximize the range of motion until maximum improvement was achieved.

Follow-up examinations were done (as per the usual routine) at $3,6,12$, and 24 months from surgery. The collected data were compared to those from the last examination. X-ray and final clinical examinations were performed at the two-year follow-up. The radiographic classification of arthritis developed by Samilson and Prieto 
Table 1 Pre- and postoperative constant scores at the final follow-up

\begin{tabular}{|c|c|c|c|}
\hline Patient & $\begin{array}{l}\text { Preoperative } \\
\text { constant score }\end{array}$ & $\begin{array}{l}\text { Postoperative } \\
\text { constant score }\end{array}$ & $\begin{array}{l}\text { Increase } \\
\text { in score }\end{array}$ \\
\hline 1 & 52 & 74 & 22 \\
\hline 2 & 48 & 85 & 37 \\
\hline 3 & 61 & 84 & 23 \\
\hline 4 & 31 & 75 & 44 \\
\hline 5 & 38 & 80 & 42 \\
\hline 6 & 41 & 84 & 43 \\
\hline 7 & 24 & 79 & 55 \\
\hline 8 & 46 & 75 & 29 \\
\hline 9 & 54 & 89 & 35 \\
\hline 10 & 32 & 81 & 49 \\
\hline 11 & 60 & 75 & 15 \\
\hline 12 & 31 & 78 & 47 \\
\hline 13 & 38 & 86 & 48 \\
\hline 14 & 43 & 70 & 27 \\
\hline 15 & 36 & 68 & 32 \\
\hline 16 & 38 & 74 & 36 \\
\hline 17 & 47 & 78 & 31 \\
\hline 18 & 28 & 91 & 63 \\
\hline 19 & 36 & 79 & 43 \\
\hline 20 & 38 & 84 & 46 \\
\hline 21 & 41 & 86 & 45 \\
\hline 22 & 45 & 73 & 28 \\
\hline 23 & 57 & 61 & 4 \\
\hline 24 & 60 & 88 & 28 \\
\hline 25 & 58 & 87 & 29 \\
\hline 26 & 31 & 68 & 37 \\
\hline 27 & 46 & 85 & 39 \\
\hline 28 & 53 & 83 & 30 \\
\hline 29 & 41 & 89 & 48 \\
\hline 30 & 49 & 78 & 29 \\
\hline 31 & 55 & 52 & -3 \\
\hline 32 & 45 & 70 & 25 \\
\hline 33 & 35 & 86 & 51 \\
\hline 34 & 69 & 79 & 10 \\
\hline 35 & 56 & 88 & 32 \\
\hline 36 & 37 & 83 & 46 \\
\hline 37 & 29 & 72 & 43 \\
\hline 38 & 37 & 41 & 4 \\
\hline 39 & 36 & 85 & 49 \\
\hline 40 & 41 & 79 & 38 \\
\hline 41 & 55 & 84 & 29 \\
\hline 42 & 57 & 89 & 32 \\
\hline 43 & 42 & 85 & 43 \\
\hline 44 & 31 & 77 & 46 \\
\hline 45 & 20 & 86 & 66 \\
\hline 46 & 47 & 86 & 39 \\
\hline 47 & 65 & 89 & 24 \\
\hline Mean & 43.8 & 79.1 & 35.3 \\
\hline
\end{tabular}

Table 1 continued

\begin{tabular}{llll}
\hline Patient & $\begin{array}{l}\text { Preoperative } \\
\text { constant score }\end{array}$ & $\begin{array}{l}\text { Postoperative } \\
\text { constant score }\end{array}$ & $\begin{array}{l}\text { Increase } \\
\text { in score }\end{array}$ \\
\hline SD & 12.9 & 14.9 & 15.1 \\
Max & 69 & 91 & 66 \\
Min & 20 & 41 & -3 \\
\hline
\end{tabular}

[26] was used to follow arthritic changes in the shoulder from preoperative to final follow-up radiographs.

Statistical methods

Statistical analysis was performed with the Intercooled Stata 9.0 software package for Windows (Stata Corporation, College Station, TX, USA). A logistic regression model was developed to investigate the influences of the selected factors on outcome score (dependent variable). Variables were eligible for incorporation into the model if they were significantly $(p<0.05)$ associated with a positive trend in the outcome. The variables examined as potential predictors (independent factors) were patient age, gender, pre- and postoperative loss of forward elevation, pre- and postoperative loss of external rotation with the arm at the side, pre- and postoperative distance between the glenoid and the humeral head on the axillary X-ray, type of glenoid cartilage lesion [small $\left(<2 \mathrm{~cm}^{2}\right)$, large $\left(>2 \mathrm{~cm}^{2}\right)$, or total (all of the surface of the glenoid), position of the glenoid cartilage lesion (anterior, posterior, or center), use of an engineered hyaluronic acid membrane. These variables were considered to be dichotomous (value: 0/1). The relationship between each factor and increase in outcome score was tested with the $\chi^{2}$ test (bivariate analysis). The Pearson correlation coefficient (PCC) was employed to assess the interobserver reliability for outcome and arthritis classification, as evaluated by three different observers.

\section{Results}

The mean Constant score of the two groups at the time of operation was 43.8 points (SD 12.9). At two years of follow-up, the mean Constant score had reached 79.1 points (SD 14.9; $p<0.05$ ) (Table 1).

The mean SST score changed from 4.9 points (SD 1.8) to 9.4 points (SD 1.9) (Table 2). The PCC was close to 1 (0.9143), indicating the low variability in the outcome measurement.

There were no statistical differences among the three different observers in the PCC analysis $(p<0.05)$ of the outcome scores. 
Table 2 Pre- and postoperative SST scores at the final follow-up

\begin{tabular}{|c|c|c|c|}
\hline Patients & Preoperative SST & Postoperative SST & Increase in SST \\
\hline 1 & 8 & 9 & 1 \\
\hline 2 & 4 & 11 & 7 \\
\hline 3 & 3 & 12 & 9 \\
\hline 4 & 6 & 10 & 4 \\
\hline 5 & 5 & 9 & 4 \\
\hline 6 & 4 & 7 & 3 \\
\hline 7 & 5 & 8 & 3 \\
\hline 8 & 6 & 9 & 3 \\
\hline 9 & 7 & 10 & 3 \\
\hline 10 & 3 & 11 & 8 \\
\hline 11 & 4 & 9 & 5 \\
\hline 12 & 5 & 12 & 7 \\
\hline 13 & 5 & 9 & 4 \\
\hline 14 & 6 & 8 & 2 \\
\hline 15 & 3 & 9 & 6 \\
\hline 16 & 7 & 11 & 4 \\
\hline 17 & 6 & 9 & 3 \\
\hline 18 & 5 & 10 & 5 \\
\hline 19 & 6 & 12 & 6 \\
\hline 20 & 7 & 12 & 5 \\
\hline 21 & 4 & 10 & 6 \\
\hline 22 & 3 & 11 & 8 \\
\hline 23 & 2 & 3 & 1 \\
\hline 24 & 4 & 8 & 4 \\
\hline 25 & 5 & 9 & 4 \\
\hline 26 & 4 & 11 & 7 \\
\hline 27 & 6 & 10 & 4 \\
\hline 28 & 4 & 10 & 6 \\
\hline 29 & 7 & 7 & 0 \\
\hline 30 & 5 & 8 & 3 \\
\hline 31 & 5 & 5 & 0 \\
\hline 32 & 3 & 8 & 5 \\
\hline 33 & 3 & 10 & 7 \\
\hline 34 & 8 & 11 & 3 \\
\hline 35 & 3 & 9 & 6 \\
\hline 36 & 4 & 8 & 4 \\
\hline 37 & 5 & 9 & 4 \\
\hline 38 & 2 & 3 & 1 \\
\hline 39 & 6 & 11 & 5 \\
\hline 40 & 5 & 10 & 5 \\
\hline 41 & 6 & 9 & 3 \\
\hline 42 & 7 & 8 & 1 \\
\hline 43 & 2 & 9 & 7 \\
\hline 44 & 4 & 8 & 4 \\
\hline 45 & 7 & 9 & 2 \\
\hline 46 & 8 & 8 & 0 \\
\hline 47 & 5 & 8 & 3 \\
\hline Mean & 4.9 & 9.1 & 4.1 \\
\hline
\end{tabular}

Table 2 continued

\begin{tabular}{llcl}
\hline Patients & Preoperative SST & Postoperative SST & Increase in SST \\
\hline SD & 1 & 2.3 & 2.3 \\
Max & 8 & 12 & 9 \\
Min & 2 & 3 & 0
\end{tabular}

Table 3 Variate analysis (relationships of variables to increases in the Constant and SST scores)

\begin{tabular}{ll}
\hline Variable & $p\left(\chi^{2}\right.$ test $)$ \\
\hline Patient age & 0.91 \\
Male & 0.83 \\
Female & 0.86 \\
Increase in forward elevation & 0.04 \\
Increase in external rotation & 0.03 \\
Glenohumeral distance & 0.91 \\
Glenoid cartilage lesion type & \\
Small & 0.03 \\
Large & 0.49 \\
Total & 0.75 \\
Position of glenoid cartilage lesion & \\
Anterior & 0.16 \\
Posterior & 0.25 \\
Centered & 0.01 \\
Use of engineered hyaluronic acid membrane & 0.54 \\
\hline
\end{tabular}

Twenty-one patients $(87.5 \%)$ had good outcomes. Three patients $(12.5 \%)$ had poor outcomes that were related to the progression of arthritis to Samilson-Prieto III and a squared humeral head (patient nos. 23, 31, and 38).

No statistical differences were found at X-ray examination between the pre- and postoperative glenohumeral distances: it remained a mean of $2.4 \mathrm{~mm}$ (range 1-4 mm, SD 1.60) $(p>0.05)($ Table 3$)$.

Age and sex were not related to outcome $(p>0.05$; Table 3).

When the results were stratified, we found that small $\left(<2 \mathrm{~cm}^{2}\right)$ and centered glenoid lesions $(p<0.05)$ gave better clinical scores, while treatment with an engineered hyaluronic acid membrane had no affect on the final outcome $(p>0.05)$; (Table 3). Patients with involvement of the whole glenoid surface had the poorest outcomes $(p>0.05$; Table 3).

\section{Discussion}

Arthroscopy allows joint irrigation with removal of cartilage debris, cytokines, and inflammatory mediators [10]. 
Arthroscopic debidement associated with capsular release may provide significant pain relief and improve ROM in patents with capsular contracture of $>15^{\circ}$ [31]. Just as other research findings have shown how osteochondral lesions of $>2 \mathrm{~cm}^{2}$ are correlated with persistent pain as a predictive variable for the ultimate failure of the arthroscopic procedure [31], the patients with the worst outcomes in our study were those with large cartilaginous defects. Severe arthritis does not seem to be usefully treated with arthroscopy because of deteriorating outcomes over time and poor functional results [32, 33]. Shoulder arthritis is followed by progressive restriction of ROM due to the contracture of the capsule and deformity of the humeral head [16]. For a peripheral cartilage lesion, the restriction of the glenohumeral joint volume, the compression of the damaged glenoid cartilage surface, the pivot mechanism, and the eccentric loads can all promote squaring of the humeral head [12]. $360^{\circ}$ capsulotomy reduces compression between the humeral head and the glenoid and can therefore lead to an improvement in the ROM. This procedure is mandatory in all cases involving an arthroscopic approach to stiff arthritic joints. Patients affected by degenerative joint diseases with residual joint space can improve shoulder function and obtain pain relief after arthroscopic debridement. The unchanged glenohumeral distance indicates that the arthritic process is stable, and biological resurfacing of the glenoid with an engineered hyaluronic acid membrane does not appear to lead to better outcomes than debridement and capsulotomy.

In a young, active person with a focal symptomatic chondral lesion, arthroscopic approach with capsulotomy, debridement, and microfractures could be a plausible option to achieve a good outcome and (probably) delay arthritic evolution. In cases with large lesions, the arthroscopic approach appears to give fair outcomes and a deterioration over time. The effects of arthroscopic debridement in cases of degenerative shoulder disease have been explored by Van Thiel et al. [32], who reported favorable results on pain relief and recovery of shoulder function in 55 out of 81 selected patients at an average follow-up of 27 months, even if there are some notable differences between our study and that of van Thiel et al. [32], such as a lower grade of arthritis.

Who are the best candidates for arthroscopy in shoulder osteoarthritis? Based on the results of the current study, young men aged 30-55 years old with a small, centered glenoid cartilage lesion and a mild loss of ROM should benefit from this treatment. Data collected in this study cannot guarantee a certain perspective in patients arthroscopically managed for early shoulder osteoarthritis. We need more long-term follow-up data, a large case series, and a histological evaluation of second-look cases before considering the procedures described in this paper as reliable and safe.
The study has numerous limitations: (1) the lack of a control group; (2) various kinds of articular lesion were treated; (3) the lack of postoperative MRI control group; (4) the lack of an arthroscopic second look allowing the histological analysis of the soft tissue, which could resolve the issue of the difference between the normal fibrocartilage created after implementing microfractures and the features of the fibrocartilage grown on a scaffold of hyaluronic acid membrane. Given the aforementioned limitations, the main findings of this work are that progression of symptomatic arthritis was seen in only $12.5 \%$ of the patients (three cases), and that the soft tissue procedures (capsulectomy and synovectomy) associated with microfractures are suitable for use in this type of patient. Arthroscopic capsular release delays disease progression by reducing load forces and improving ROM and joint elasticity.

\section{Conflict of interest None.}

Open Access This article is distributed under the terms of the Creative Commons Attribution License which permits any use, distribution, and reproduction in any medium, provided the original author(s) and the source are credited.

\section{References}

1. Cameron ML, Kocher MS, Briggs KK, Horan MP, Hawkins RJ (2003) The prevalence of glenohumeral osteoarthrosis in unstable shoulders. Am J Sports Med 31(1):53-55

2. Gartsman GM, Taverna E (1997) The incidence of glenohumeral joint abnormalities associated with full-thickness, reparable rotator cuff tears. Arthroscopy 13(4):450-455

3. Cole BJ, Yanke A, Provencher MT (2007) Nonarthroplasty alternatives for the treatment of glenohumeral arthritis. J Shoulder Elbow Surg 16(5 Suppl):S231-S240

4. Warner JJ, Bowen MK, Deng XH, Hannafin JA, Arnoczky SP, Warren RF (1998) Articular contact patterns of the normal glenohumeral joint. J Shoulder Elbow Surg 7(4):381-388

5. Meyer DC, Fucentese SF, Koller B, Gerber C (2004) Association of osteopenia of the humeral head with full-thickness rotator cuff tears. J Shoulder Elbow Surg 13(3):333-337

6. Elser F, Braun S, Dewing CB, Millett PJ (2010) Glenohumeral joint preservation: current options for managing articular cartilage lesions in young, active patients. Arthroscopy 26(5):685-696

7. Stone JL, Beaupre GS, Hayes WC (1983) Multiaxial strength characteristics of trabecular bone. J Biomech 16(9):743-752

8. van der Helm FC (1994) Analysis of the kinematic and dynamic behavior of the shoulder mechanism. J Biomech 27(5):527-550

9. Lehtinen JT, Tingart MJ, Apreleva M, Warner JJ (2004) Total, trabecular, and cortical bone mineral density in different regions of the glenoid. J Shoulder Elbow Surg 13(3):344-348

10. Frich LH, Odgaard A, Dalstra M (1998) Glenoid bone architecture. J Shoulder Elbow Surg 7(4):356-361

11. McCarty LP 3rd, Cole BJ (2005) Nonarthroplasty treatment of glenohumeral cartilage lesions. Arthroscopy 21(9):1131-1142

12. Savoie FH 3rd, Brislin KJ, Argo D (2009) Arthroscopic glenoid resurfacing as a surgical treatment for glenohumeral arthritis in the young patient: midterm results. Arthroscopy 25(8):864-871 
13. Dines JS, Strauss EJ, Fealy S, Craig EV (2007) Arthroscopicassisted core decompression of the humeral head. Arthroscopy 23(1):103.e1-103.e4

14. Noël E, Hardy P, Hagena FW, Laprelle E, Goebel F, Faure C, Favard L, Gaudin P, Christ R, Baudot C, Dietl J, Goupille P (2009) Efficacy and safety of Hylan G-F 20 in shoulder osteoarthritis with an intact rotator cuff. Open-label prospective multicenter study. Joint Bone Spine 76(6):670-673

15. Merolla G, Sperling JW, Paladini P, Porcellini G (2011) Efficacy of hylan G-F 20 versus 6-methylprednisolone acetate in primary glenohumeral osteoarthritis: a retrospective controlled trial. Musculoskeletal Surg 95:215-224

16. Weinstein DM, Bucchieri JS, Pollock RG, Flatow EL, Bigliani LU (2000) Arthroscopic debridement of the shoulder for osteoarthritis. Arthroscopy 16(5):471-476

17. Ho JY, Miller SL (2007) Allografts in the treatment of athletic injuries of the shoulder. Sports Med Arthrose 15(3):149-157

18. Scheibel M, Bartl C, Magosch P, Lichtenberg S, Habermeyer P (2004) Osteochondral autologous transplantation for the treatment of full-thickness articular cartilage defects of the shoulder. J Bone Joint Surg Br 86:991-997

19. Lollino N, Pellegrini A, Paladini P, Campi F, Porcellini G (2011) Gleno-humeral arthritis in young patients: clinical and radiographic analysis of humerus resurfacing prosthesis and meniscus interposition. Musculoskelet Surg 95(Suppl 1):S59-S63

20. Sperling JW, Steinmann SP, Cordasco FA, Henshaw DR, Coons DA, Burkhead WZ (2006) Shoulder arthritis in the young adult: arthroscopy to arthroplasty. Instr Course Lect 55:67-74

21. Millett PJ, Huffard BH, Horan MP, Hawkins RJ, Steadman JR (2009) Outcomes of full-thickness articular cartilage injuries of the shoulder treated with micro fracture. Arthroscopy 25(8):856863

22. Siebold R, Lichtenberg S, Habermeyer P (2003) Combination of micro fracture and periostal-flap for the treatment of focal full thickness articular cartilage lesions of the shoulder: a prospective study. Knee Surg Sports Traumatol Arthrosc 11:183-189
23. Steadman JR, Rodkey WG, Rodrigo JJ (2001) Microfracture: surgical technique and rehabilitation to treat chondral defects. Clin Orthop Relat Res Oct(Suppl 391):S362-S369

24. Strauss E, Schachter A, Frenkel S, Rosen J (2009) The efficacy of intra-articular hyaluronan injection after the micro fracture technique for the treatment of articular cartilage lesions. Am J Sports Med 37(4):720-726

25. Gobbi A, Kon E, Berruto M, Francisco R, Filardo G, Marcacci M (2006) Patellofemoral full-thickness chondral defects treated with Hyalograft-C: a clinical, arthroscopic, and histologic review. Am J Sports Med 34(11):1763-1773

26. Samilson RL, Prieto V (1983) Dislocation arthropathy of the shoulder. J Bone Joint Surg Am 65(4):456-460

27. Lippitt SB, Harryman DT II, Matsen FA III (1993) A practical tool for evaluating function: the simple shoulder test. In: Matsen FA III, Fu FH, Hawkins RJ (eds) The shoulder: a balance of mobility and stability. American Academy of Orthopaedic Surgeons, Rosemont, pp 501-518

28. Constant CR, Murley AH (1987) A clinical method of functional assessment of the shoulder. Clin Orthop Relat Res 214:160-164

29. Outerbridge RE (1961) The etiology of chondromalacia patellae. J Bone Joint Surg Br 43:752-757

30. Parson IM, Millett PJ, Warner JP (2004) Glenoid wear after shoulder hemiarthroplasty: a quantitative analysis. Clin Orthop Relat Res 421:120-125

31. Cameron BD, Galatz ML, Ramsey ML, Williams GR, Iannotti JP (2002) Non-prosthetic management of grade IV osteochondral lesions of the glenohumeral joint. J Shoulder Elbow Surg 11:25-32

32. Van Thiel GS, Sheehan S, Frank RM, Slabaugh M, Cole BJ, Nicholson GP, Romeo AA, Verma NN (2010) Retrospective analysis of arthroscopic management of glenohumeral degenerative disease. Arthroscopy 26(11):1451-1455

33. Chong PY, Srikumaran U, Kuye IO, Warner JJ (2011) Glenohumeral arthritis in the young patient. J Shoulder Elbow Surg 20(Suppl 2):S30-S40. doi:10.1016/j.jse.2010.11.014 